\title{
A Panax notoginseng Root Tip Meristem Biosensor and Its Sensing Kinetics for Five Important Nitrogen Nutrients
}

\author{
Zi Qing Zheng, ${ }^{1}$ Bo Niu, ${ }^{1}$ Ding Qiang Lu $\mathbb{D}^{1,2}$ and Guang Chang Pang $\mathbb{D}^{1,2}$ \\ ${ }^{1}$ College of Biotechnology \& food Science, Tianjin University of Commerce, Tianjin 300314, China \\ ${ }^{2}$ Tianjin Key Laboratory of Food Biotechnology, Tianjin 300314, China
}

Correspondence should be addressed to Ding Qiang Lu; dqlu@tjcu.edu.cn and Guang Chang Pang; pangtjcu@163.com

Received 1 March 2021; Revised 16 April 2021; Accepted 30 May 2021; Published 30 June 2021

Academic Editor: Bruno C. Janegitz

Copyright (C) 2021 Zi Qing Zheng et al. This is an open access article distributed under the Creative Commons Attribution License, which permits unrestricted use, distribution, and reproduction in any medium, provided the original work is properly cited.

\begin{abstract}
Plants absorb nitrogen mainly through their roots. Nitrogen sensing is required for the absorption and transport of different nitrogen nutrients. In this study, we constructed biosensors with immobilized Panax notoginseng root tip meristems based on a three-electrode system and successfully determined the kinetics of the interactions between the P. notoginseng root tip meristems and five important nitrogen nutrients, namely, urea, sodium nitrate, sodium glutamate, disodium inosinate, and disodium guanylate. We discovered that the biosensor's sensing kinetics was similar to the enzyme-substrate kinetics, and the receptor-ligand interconnected allosteric interaction constant $\mathrm{Ka}(\mathrm{mol} / \mathrm{L})$, analogous to the Michaelis constant, was calculated. The result showed that the root tip meristems of two- to four-year-old $P$. notoginseng plants had a higher capacity to sense inorganic nitrogen nutrients (sodium nitrate and urea) than the three organic nitrogen nutrients. The ability of the plants to sense inorganic nitrogen nutrients decreased with an increase in plant age. The sensing sensitivity of four-year-old $P$. notoginseng plants to disodium inosinate and disodium guanylate was 100- to 10,000-fold lower than that of the two- and threeyear-old plants. Additionally, the capability to sense sodium glutamate decreased initially and then increased with an increase in plant age. The biosensors reached an ultra-sensitive level $\left(1 \times 10^{-22} \mathrm{~mol} / \mathrm{L}\right)$ in sensing the five nitrogen nutrients and exhibited advantages such as good stability and reproducibility, low cost, a simple structure, and a rapid response, providing a new approach for quantitative determination of the capability of plants to sense different nitrogen nutrients.
\end{abstract}

\section{Introduction}

Nitrogen nutrition is closely related to plant growth [1]. Botanists have made significant progress in understanding the absorption and transport of nitrate nitrogen, ammonium nitrogen, and amino acid nitrogen in plants. For instance, nitrate transceptors (NRT) NRT1.1 and NRT2.1 [2-5] as well as ammonium transporters (AMT) AMT1.1 and AMT1.3 [6, 7] were identified in the root system of the model plant Arabidopsis thaliana. Amino acid permease (AAP2) and lysine/histidine transporter (LHT1) were speculated to be amino acid transporters $[8,9]$, and L-type lectin receptor kinase $\mathrm{P} 2 \mathrm{~K} 1$ is an essential receptor for monitoring extracellular purine (ATP) in A. thaliana [10]. Glutamate receptors (GLRs) are related to defense signals in A. thaliana [11, 12]. Most of these studies focused on the transport of nitrogen nutrients and signal transduction in plants. However, plants must sense the nitrogen nutrient status in the environment by their roots first and then absorb and use the nitrogen according to their own requirements [13-16]. In other words, sensing is the prerequisite for absorption. Therefore, it is relevant to study the nitrogen-sensing receptors of plants, assess the mechanisms of nitrogen absorption and transport, and quantify the capacity of plants to sense different nitrogen nutrients. However, plant extracellular sensing, the pathways of intracellular signal amplification and transduction, and nitrogen sensing receptor-related quantification methods have been seldom reported so far.

Nitrate is the main form of inorganic nitrogen available to plants and is used as a nitrogen source as well as an important signal that regulates the growth and development of plant organs [17]. Urea, which is currently the most commonly used inorganic nitrogen fertilizer, plays an important role in transamination, deamination, the ornithine cycle, 
and the synthesis of arginine and nucleotides in higher plants and animals, especially in higher animals. However, the sensing and absorption of inorganic nitrogen by roots must be coordinated and balanced with the needs for amino acids, nucleotides, and the carbon skeleton synthesized through the Calvin cycle after carbon dioxide is fixed in leaf chloroplasts during photosynthesis. Subsequently, nitrogen is used in the synthesis of proteins or DNA replication. Thus, inorganic nitrogen is useful only when plants are in need of nitrogen, whereas excessive inorganic nitrogen nutrients cause toxicity and side effects [18]. As the most important intermediate metabolite in amino acid metabolism, sodium glutamate forms an "amino pool" consisting of glutamine and asparagine through the catalysis of alanine aminotransferase and aspartate aminotransferase. Disodium inosinate is the most important intermediate product in nucleotide metabolism. Disodium guanylate can be decomposed from GTP into GDP, providing energy for protein synthesis, G-protein signal amplification, and the transduction, kinase activation, and transport of various proteins, nucleic acids, and transcription factors inside and outside the nucleus. Similar to sodium glutamate, the sensing receptor of disodium guanylate is also T1R1, but their binding sites are in different domains [19]. We selected sodium nitrate, urea, sodium glutamate, disodium inosinate, and disodium guanylate as ligands to determine the sensing capability of plants, and the results were compared.

In our previous study, we successfully constructed tussah antennae and receptor biosensors to sense a male Bombyx mori sex attractant pheromone (bombykol) as well as other higher animal tissue or receptor biosensors to sense umami, sweet, bitter, and oleogustus tastes [20]. Those receptors belong to the G-protein-coupled receptor (GPCR) superfamily. The umami receptor essentially transmits a nitrogen signal [21]. Researchers have become increasingly aware that the GPCR exists widely among various cells, tissues, and organs of animals, plants, and microorganisms, playing an important role in the sensing of multiple types of nutrients [22].

In the current study, we extended the tissue sensor technology to the root tissue of Panax notoginseng (Burk.) FH Chen, a perennial herbaceous medicinal plant belonging to the Araliaceae family. The number of available varieties of $P$. notoginseng is very limited. The plants are sensitive to nitrogen during growth and development [23]. Improper use of nitrogen fertilizer not only wastes resources but also hinders biomass accumulation in the roots, stems, and leaves and increases rust spots, root rot, and the mortality of $P$. notoginseng plants [24], which is aggravated by consecutive monoculture. Previous studies have mainly focused on the effects of nitrogen fertilizer input on the agronomic traits, yield, and growth of $P$. notoginseng. However, its preference for particular nitrogen fertilizers is still inconclusive, and its nitrogen-sensing mechanism is unclear. Therefore, the intensive cultivation and variety improvement of $P$. notoginseng are restricted. Quantification of the "preference" of P. notoginseng for particular nitrogen forms would benefit the sustainable cultivation of $P$. notoginseng and reduce nitrogen fertilizer inputs. We constructed a root tip meristem biosensor using plants of different ages and analyzed the kinetics of the $P$. notoginseng root tip meristem in sensing the five types of important nitrogen forms described above.

\section{Materials and Methods}

2.1. Materials and Reagents. Two-, three-, and four-year-old fresh $P$. notoginseng plants (10 plants were selected from each age group) were collected from Wenshan Prefecture, Yunnan Province. The reagents and other materials used in this study included soluble starch, anhydrous $\mathrm{CaCl}_{2}$, urea, and sodium nitrate (Tianjin Yingda Rare Chemical Reagent Factory, China); sodium alginate, glutaraldehyde, and $\mathrm{K}_{3} \mathrm{Fe}(\mathrm{CN})_{6}$ (Tianjin Bodie Chemical, China); sodium glutamate, disodium inosinate, and disodium guanylate (Shanghai Meryer Chemical Technology, China); nucleopore membranes with a pore diameter of $0.22 \mu \mathrm{m}$ and circumference of $25 \mathrm{~mm}$ (Whatman, UK); and suede and $\alpha-\mathrm{Al}_{2} \mathrm{O}_{3}$ particles with diameters of $1.00,0.30$, and $0.05 \mu \mathrm{m}$ (Shanghai Chenhua Instrument, China). The reagents used were all analytical grade. Ultrapure water was used in the experiments.

2.2. Instruments. The instruments and equipment used in this study included an analytical balance (Shanghai Precision \& Scientific Instrument, China); CHI660E electrochemical workstation and three-electrode system including a glassy carbon electrode, $\mathrm{Ag} / \mathrm{AgCl}$ electrode, and platinum wire electrode (Shanghai Chenhua Instrument, China); KQ 3200B ultrasonic cleaner (Kunshan Ultrasonic Instrument, China); and Milli-Q Reference Ultrapure Water System (EMD Millipore, USA).

\subsection{Methods}

2.3.1. Electrode Pretreatment and Characterization. The glassy carbon electrode was polished with aluminum $(\alpha-$ $\mathrm{Al}_{2} \mathrm{O}_{3}$ ) powder of different particle sizes (1.0, 0.3, and 0.05 $\mu \mathrm{m}$ sequentially) on a piece of moistened suede and cleaned in an ultrasonic water bath for $30 \mathrm{~s}$ after each polish. This procedure was repeated 2-3 times. Following this, the glassy carbon electrode was cleaned with ultrapure water. The electrode was placed in a $1 \mathrm{~mol} / \mathrm{L} \mathrm{H}_{2} \mathrm{SO}_{4}$ solution and activated by means of cyclic voltammetry, scanning from -1.0 to 1.0 $\mathrm{V}$ at a rate of $100 \mathrm{mV} / \mathrm{s}$. The activated glassy carbon electrode was placed in a $1 \times 10^{-3} \mathrm{~mol} / \mathrm{L} \mathrm{K}_{3} \mathrm{Fe}(\mathrm{CN})_{6}$ solution containing $0.20 \mathrm{~mol} / \mathrm{L} \mathrm{KNO}_{3}$ and was characterized using cyclic voltammetry, scanning from -0.1 to $0.6 \mathrm{~V}$ at a rate of $50 \mathrm{mV} / \mathrm{s}$. The potential difference peak of the cyclic voltametric curve of the pretreated glassy carbon electrode was less than 80 $\mathrm{mV}$, indicating that the electrode was usable. The characterized electrode was cleaned with ultrapure water and then dried under nitrogen gas for later use.

2.3.2. Preparation of the Plant Root Tip Meristem Immobilizer. One gram of soluble starch was weighed and dissolved in $99 \mathrm{~mL}$ ultrapure water, and $1 \mathrm{~mL}$ of $10 \%$ glutaraldehyde was added. After being heated with constant stirring in a water bath at $80^{\circ} \mathrm{C}$ for $30 \mathrm{~min}$, the solution was incubated overnight at room temperature to enable full crosslinking between starch and glutaraldehyde to form a $1 \%$ soluble aldehyde starch solution. Two grams of sodium 
alginate was placed in $100 \mathrm{~mL}$ ultrapure water and then heated with stirring in a water bath until the sodium alginate was completely dissolved to form a $2 \%$ sodium alginate solution. Five grams of anhydrous $\mathrm{CaCl}_{2}$ was dissolved in $100 \mathrm{~mL}$ ultrapure water to obtain a $5 \% \mathrm{CaCl}_{2}$ solution.

2.3.3. Construction of Biosensors with Immobilized $P$. notoginseng Root Tip Meristems. The fresh roots of twoyear-old $P$. notoginseng plants were cleaned with ultrapure water. After removal of the water remaining on the root surface, root tips approximately $1 \mathrm{~cm}$ long from the lateral roots, including the root hairs, were collected using a scalpel blade and then minced in a clean petri dish. A small amount of minced $P$. notoginseng root tip meristem tissue was placed on the center of a piece of nucleoporous membrane, covering an area of approximately $0.25 \mathrm{~cm}^{2}$. Soluble starch and sodium alginate solutions were mixed at a 1:1 ratio, and $30 \mu \mathrm{L}$ of the mixed solution was spread onto the minced root tip tissues to immobilize them, after which they were covered with another piece of nucleoporous membrane. The edge of the membranes was sealed with the mixed solution. The membranes were then immersed in $\mathrm{CaCl}_{2}$ solution, enabling a complete crosslinking between the sodium alginate and $\mathrm{CaCl}_{2}$ to form a sandwich-like detection membrane. The membranes were rinsed with ultrapure water to remove residues of $\mathrm{Cl}^{-}$and $\mathrm{Ca}^{2+}$, and then the $P$. notoginseng root tip meristems between the membranes were used to completely cover the core of a glassy carbon electrode and were fixed with a rubber band. Finally, the prepared biosensor was flushed with ultrapure water and was ready to be used.

2.3.4. Measurement Method and Data Processing. The threeelectrode system of the CHI660E electrochemical workstation was used in this study. The glassy carbon electrode wrapped with $P$. notoginseng root tip meristems supported by the membranes was used as the working electrode. The $\mathrm{Ag} / \mathrm{AgCl}$ electrode was the reference electrode, the platinum wire electrode was used as the counter electrode, and ultrapure water was used as a blank control. Under the optimized potential (Figure S1 in support materials), the response current values of the $P$. notoginseng root tip meristems interacting with the five nitrogen forms were measured using the current-time measurement method, and the measurement was performed three times for each nutrient concentration. The change rate of response current $\Delta I$ was used as the detection index. The principal of measurement is shown in Figure 1. The change rate of the response current was calculated according to the equation below.

$$
\Delta I / \%=\frac{I_{1}-I_{2}}{I_{1}} \times 100 \Delta I / \%=\frac{I_{1}-I_{2}}{I_{1}} \times 100 .
$$

Here, $I_{1}$ represents the response current of the blank control, and $I_{2}$ represents the response current of the solution to be measured.

\section{Results and Discussion}

Compared with a nanogold receptor sensor [25], which has the advantage that the effect truly caused by the receptor can be exactly known, the biosensor prepared using the root tip meristem could reflect not only the allostery resulting from the interaction between the extracellular domain of the receptor and the nitrogen nutrient ligand but also the change in the on/off state of the ion channels in the cell membrane caused by the intracellular signal transduction and cascade amplification after the binding of the receptor and ligand. In order to eliminate interferences and ensure that the measured electrochemical signal output by the root meristem was caused by the recognition and stimulation of the ligands (different nitrogen forms), we verified the signals by characterizing each of the links in the biosensor assembly.

\subsection{Electrochemical Characterization of the Electrode} Pretreatment Effect and Assembly Process. $\mathrm{H}_{2} \mathrm{SO}_{4}$ solution $(1 \mathrm{~mol} / \mathrm{L})$ was used to activate the glassy carbon electrode (GCE) and to generate negatively charged oxygencontaining groups (such as hydroxyl and carboxyl groups) on its surface to form a porous structure that had an increased effective surface area [26]. The activated glassy carbon electrode was characterized by means of cyclic voltammetry in $\mathrm{K}_{3} \mathrm{Fe}(\mathrm{CN})_{6}$ solution. Figure 2 shows the characterization result of the pretreated glassy carbon electrode. The potential difference between the oxidation peak and the reduction peak in Figure 2(a) was less than $80 \mathrm{mV}$ [25, 27], and the peak current ratio approached 1. Figure 2(b) shows that after pretreatment, the reduction and oxidation current peak of the electrode had a good linear relationship with the square root of the scan rate, indicating that the glassy carbon electrode was influenced only by the diffusion conditions, and the pretreatment was up to standard. We could thus proceed to the next assembly step.

In this study, sodium alginate-starch gel and calcium chloride were used to immobilize the $P$. notoginseng root tip meristem [27]. Immobilization of the $P$. notoginseng root tip meristem on the electrode surface was crucial in the determination of sensor kinetics. Generally, cells, tissues, or enzymes are immobilized with reagents such as calcium alginate or chitosan $[28,29]$. We fixed the $P$. notoginseng root tip meristem, which immobilized with sodium alginate and starch solution, in between two pieces of nucleoporous membranes (PVDF), to make a "sandwich" structure, and then it is fixed on the glassy carbon electrode to make a sensor. In Figure 3, the bare electrode plus the immobilized nucleoporous membrane (electrode + nucleoporous membrane) produced smooth and stable cyclic voltametric curves and showed higher oxidation and reduction peak currents than that of the electrode with the immobilized root tip meristem (electrode + nucleoporous membrane + root tip meristem), indicating that the nucleoporous membrane and $P$. notoginseng root tip meristem obstructed the movement of electrons to the electrode surface. This confirmed that the P. notoginseng root tip meristem was successfully fixed on the electrode. 


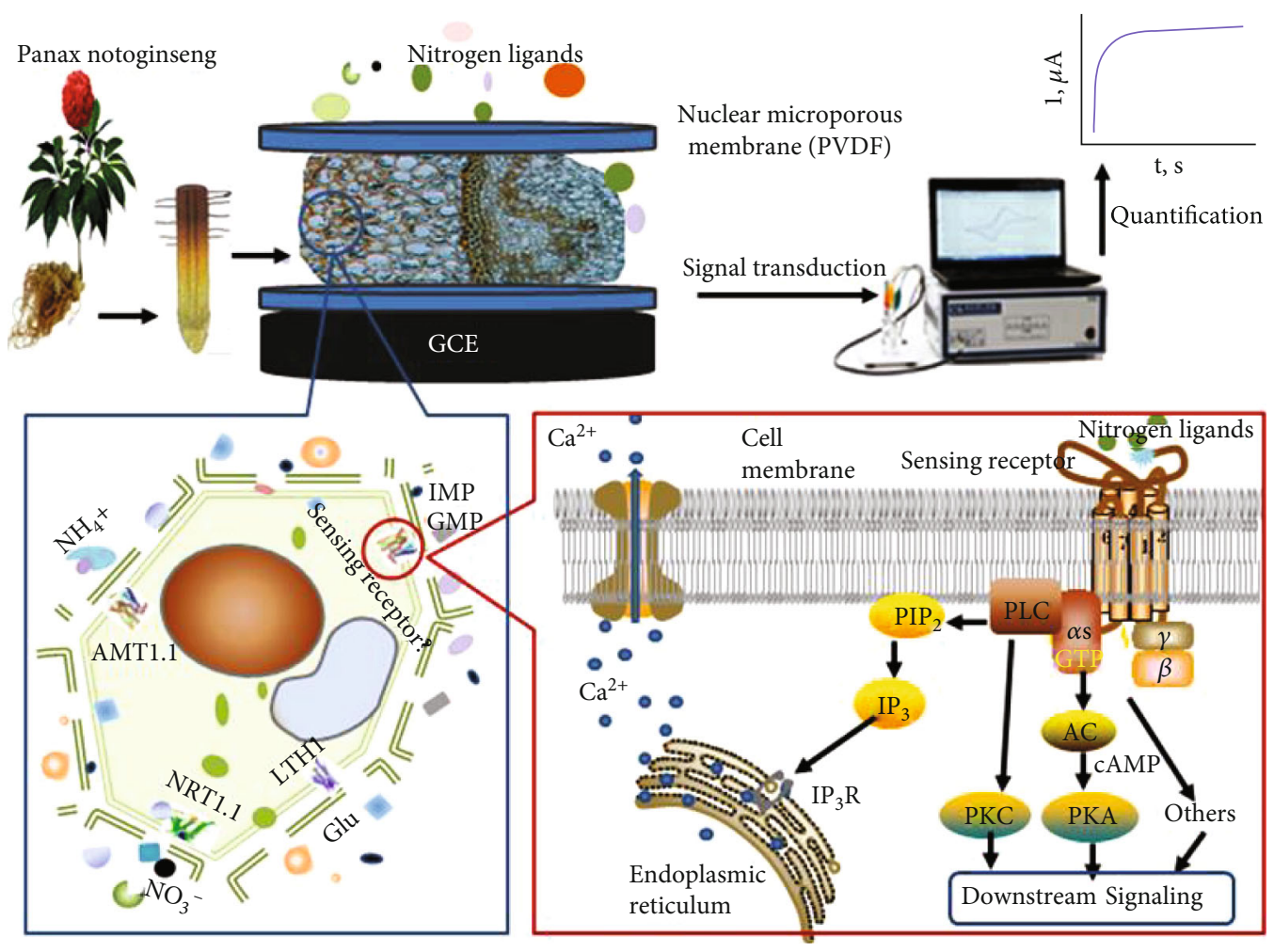

Figure 1: The measurement principle diagram of the entire assembly of the $P$. notoginseng root tip meristem biosensor. After the $P$. notoginseng root tip meristem was assembled on the electrode as a sensitive element, the five important nitrogen source ligand solutions were detected. During the detection process, the extracellular domain of the plant sensing receptor and the nitrogen source ligand allosterically interact with each other, causing the intracellular domain to change and alter the ion channel to transmit signals to the cells. The electrochemical workstation captures this nongenomic "fast pathway" signal, and the electrical signal released by the allosteric linkage between the sensing receptor and the ligand is transduced to the computer for signal recording. The sensing sensitivity of the $P$. notoginseng root tip meristem to different nitrogen source ligands can be preliminarily and quantitatively detected, and the pattern of interaction between the sensing receptor and nitrogen source ligands can be explored.

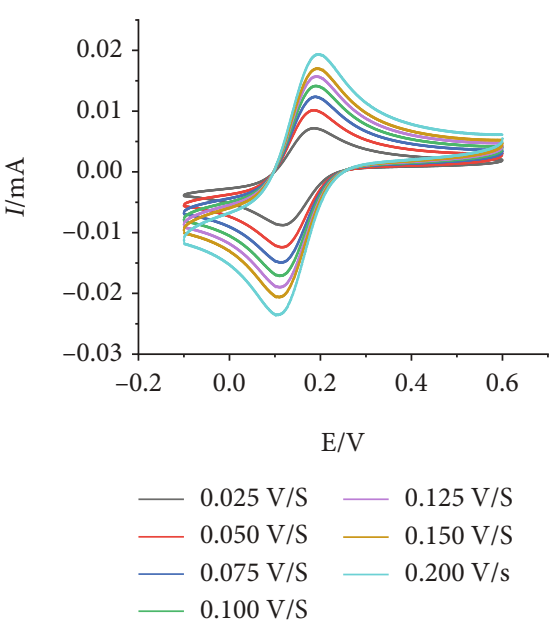

(a)

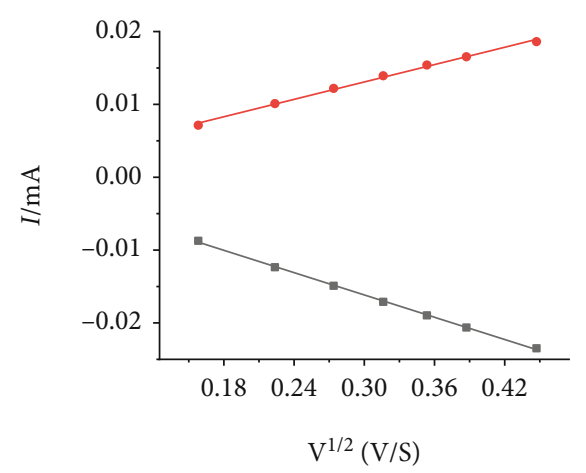

(b)

FIGURE 2: Cyclic voltammograms of the bare glassy carbon electrode (a). The scan rates were $0.025,0.050,0.075,0.100,0.125,0.150$, and 0.200 $\mathrm{V} / \mathrm{s}$. Relationship between the scan rate and the reduction and oxidation peak current of the pretreated glassy carbon electrode (b). The scan rates were $0.025,0.050,0.075,0.100,0.125,0.150$, and $0.200 \mathrm{~V} / \mathrm{s}$, and the correlation coefficients of Epa and Epc are 0.9993 and 0.9968.

\subsection{Determination of the Kinetics and Important Parameters} of the P. notoginseng Root Tip Meristem Biosensor in Sensing Five Important Nitrogen Forms. Panax notoginseng is a perennial medicinal plant. Generally, four-year-old plants have the best medicinal effects. In order to reveal the regularity of $P$. notoginseng roots in sensing nitrogen forms in 


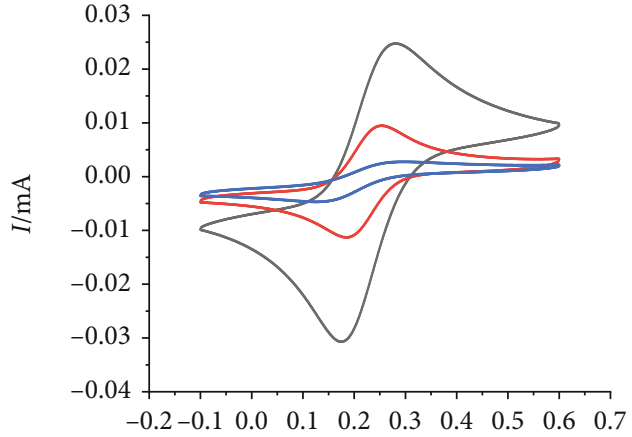

$\mathrm{E} / \mathrm{V}$

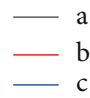

FIGURE 3: The cyclic voltametric curve of the electrodes in the assembly process. (a)-(c) represent the bare electrode, bare electrode + nuclear microporous membrane, and bare electrode + nuclear microporous membrane $+P$. notoginseng root tip meristem, respectively.

different years, we used fresh $P$. notoginseng root tip meristem tissue from two-, three-, and four-year-old plants in this study. Solutions of the five nitrogen forms with a series of concentrations ranging from $1 \times 10^{-23}$ to $1 \times 10^{-10} \mathrm{~mol} / \mathrm{L}$ were prepared. The biosensors constructed with $P$. notoginseng root tip meristems of different ages were placed sequentially from low to high concentrations in the nutrient solutions to examine detection limit using the time-current method [30]. Scans were performed at $-0.38 \mathrm{~V}$ potential and included resting for $10 \mathrm{~s}$ and then scanning for $120 \mathrm{~s}$. Based on the sensing of $P$. notoginseng to sodium nitrate in different years as an example, the minimum detectable concentrations for the biosensor prepared with two-, three-, and four-year-old plants were $1 \times 10^{-22}, 1 \times 10^{-23}$, and $1 \times$ $10^{-21} \mathrm{~mol} / \mathrm{L}$, respectively. The data were plotted using the logarithmic value of sodium nitrate concentration $\mathrm{C}(\mathrm{mol} / \mathrm{L})$ as the abscissa and the change rate of the response current $\triangle I$ as the ordinate, as shown in Figure 4. $\Delta I$ changed linearly within a certain sodium nitrate concentration range, being $10^{-22}-10^{-20}, 10^{-23}-10^{-21}$, and $10^{-21}-10^{-19} \mathrm{~mol} / \mathrm{L}$ for the biosensors prepared with two-, three-, and four-year-old $P$. notoginseng root tip meristems, respectively.

In Figure 4, the concentration ranges showing a linear relationship with $\Delta I$ were further subdivided, and the data were fitted with a hyperbolic curve using Origin2019 software to obtain the kinetic curve of the receptor-ligand interaction of the biosensors prepared with root tip meristems of different ages. The result is shown in Figure 5, and the equations obtained by hyperbolic curve fitting are shown in Table 1 . Within the low concentration range, $\Delta I$ increased linearly, and the capacity of the $P$. notoginseng root tip meristem to sense sodium nitrate was much greater than the amount of sodium nitrate in the solution. As the nitrogen concentration increased, the increase in $\Delta I$ slowed down and the curve gradually declined to a horizontal level, indicating that the receptors in the root tip meristem had reached the upper limit of their capacity to sense sodium nitrate, which was similar to the enzymatic reaction kinetics. At this time, the receptors in the root tip meristem of $P$. notoginseng were completely saturated with ligands, and the maximum electrochemical signal output was reached. In addition, biosensors prepared with $P$. notoginseng root tip meristems of different ages were used to detect the five nitrogen nutrients (urea, sodium glutamate, disodium inosinate, and disodium guanylate) at different concentrations, and the response current showed the same hyperbolic kinetic characteristics.

The $P$. notoginseng root tip meristem biosensor reflected the binding of receptors and ligands, and to a certain extent, the change in the on/off state of cell membrane ion channels caused by intracellular signal transduction and cascade amplification after the binding of receptors and ligands, which led to changes in the electrochemical signals. Since the effect of sensing and signal cascade amplification was analogous to enzymatic reaction kinetics, the receptor-ligand interaction kinetic equation could be deduced based on the enzymatic reaction kinetic equation:

$$
[E]+[S] \underset{K_{-1}}{\stackrel{K_{1}}{\longrightarrow}}[E S] \stackrel{K_{2}}{\longrightarrow} E+\operatorname{product}(P)
$$

The analogous receptor-ligand interaction kinetic equation was

$$
[R]+[L] \underset{K_{2}}{\stackrel{K_{1}}{\leftarrow}}[R L] \rightarrow \text { electric signal }\left(K_{d}\right)
$$

When the receptors in the root tip meristem were saturated, we had

$$
K_{d}=\frac{K_{2}}{K_{1}}=\frac{[R][L]}{[R L]} .
$$

If $[R T]$ was defined as the initial concentration of the receptors, then $[R]=[R T]-[R L]$; if $[L T]$ referred to the total ligand concentration, then $[L]=[L T]-[R L]$. When $[L]=[L T$ ]$-[R L]$ and $[R]=[R T]-[\mathrm{RL}]$ were substituted into equation (4), the resulting equation was as follows:

$$
[R L]^{2}-[R L]\{[R T]+[L T]+K d\}+[R T][L T]=0 .
$$

Equation (5) was transformed, and the deduced equation was

$$
\frac{1}{[R L]}=\frac{1}{[R T]}+\frac{K_{d}}{[R T]} \frac{1}{[L]}
$$

We define an important kinetic parameter $\mathrm{Ka}$ that is analogous to the Michaelis constant Km, namely, the interconnected allosteric interaction constant generated by the receptor-ligand interaction, which is the ligand concentration when half of the maximum signal output is reached [31]. The smaller the Ka value, the lower the corresponding concentration of the ligand to exert a biological effect and the higher the sensing sensitivity. A double reciprocal 


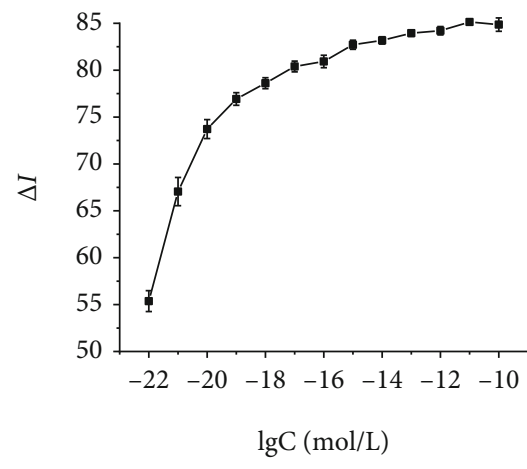

(a)

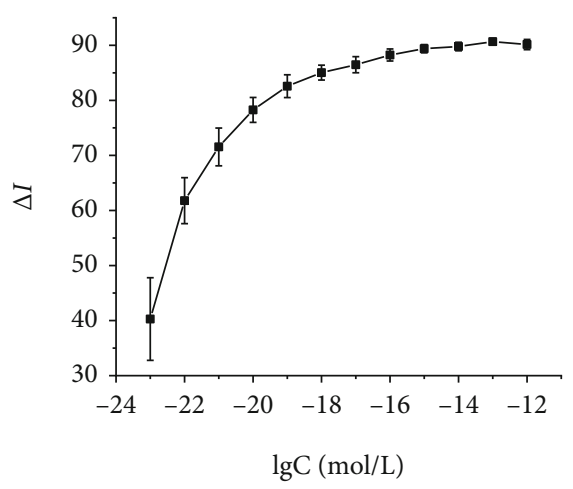

(b)

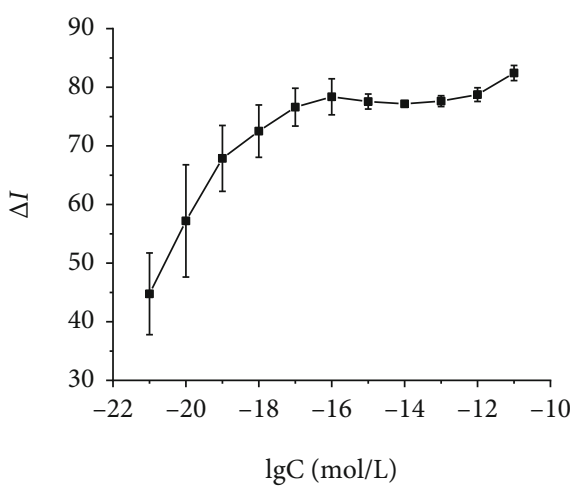

(c)

Figure 4: Current change rates of biosensors constructed with $P$. notoginseng root tip meristems of different ages in sensing sodium nitrate solutions within the set concentration range. The response current change rate in sensing sodium nitrate at $10^{-22}-10^{-11}, 10^{-23}-10^{-12}$, and $10^{-21}-10^{-10} \mathrm{~mol} / \mathrm{L}$ by using the biosensors constructed with the root tip meristems of two-year-old P. notoginseng (a), three-year-old $P$. notoginseng (b), and four-year-old P. notoginseng (c), respectively.

method was used to plot the data using the reciprocal of the mass concentration of the sodium nitrate solution as the abscissa and the reciprocal of the current change rate as the ordinate. Linear fitting was processed using Origin2019 software. The result is shown in Figure 6, and the linear fitting equations are listed in Table 2.

The linear regression equations fitted by the double reciprocal method were used to calculate the Ka values of the $P$. notoginseng root tip meristems of different ages interacting with the five important representative nitrogen nutrients, and the result is shown in Table 3 and Figure S2, S3, S4 and Table S1, S2. These results indicated that the $P$. notoginseng root tip meristem sensor provides a detection method to understand the sensing ability of the root system. Taking sodium nitrate as an example, compared with other methods for detecting sodium nitrate, this sensor has higher sensitivity, and the detected plants are more targeted, as shown in Table 4.

3.3. Kinetics of the P. notoginseng Root Tip Meristem Sensor in Sensing the Five Important Nitrogen Nutrients. The variation in the kinetic parameters of $P$. notoginseng for sensing different nitrogen forms was mainly due to the difference in its sensing sensitivity to different nutrients [35-37]. In Figure 7 , the sensing kinetic parameters of the $P$. notoginseng root system showed different trends with age, and the regu- larity in the sensing of the different nitrogen forms differed. We compared the Ka value of the biosensor constructed with root tip meristems of plants of different ages in sensing the five nitrogen forms and found that their capacity to sense inorganic nitrogen forms (nitrate nitrogen and urea) was higher than their capacity to sense organic nitrogen, such as amino acid and nucleotide-type nitrogen, and their capacity to sense sodium nitrate was higher than that of urea. Therefore, it is easier for $P$. notoginseng plants to sense and absorb nitrate than urea. However, the sensing capability of $P$. notoginseng decreased with increased age, which is obviously consistent with their decreased capability to synthesize organic nitrogen (amino acids and nucleotides) using inorganic nitrogen and carbon skeletons generated from photosynthesis when they age. Considering the overall Ka value, it is clear that the capability of the $P$. notoginseng root tip meristem to sense different nitrogen forms was closely associated with the age of the root system. The capability of four-year-old $P$. notoginseng root tip meristems to sense the five nitrogen forms was lower than that of the two-year-old plants, and their capability to sense two types of nucleotide nitrogen decreased from $9.1561 \times 10^{-21}$ to $1.3703 \times 10^{-17}$ and from $1.5147 \times 10^{-20}$ to $8.5399 \times 10^{-18} \mathrm{~mol} / \mathrm{L}$ (Figure S2, S3, S4 and Table S1, S2), indicating a 100- to 10,000-fold decrease.

The results indicated that the capability of the $P$. notoginseng root system to sense nitrogen forms was closely related 


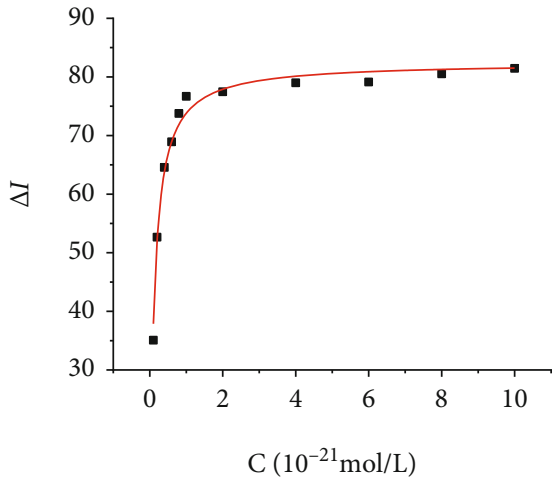

(a)

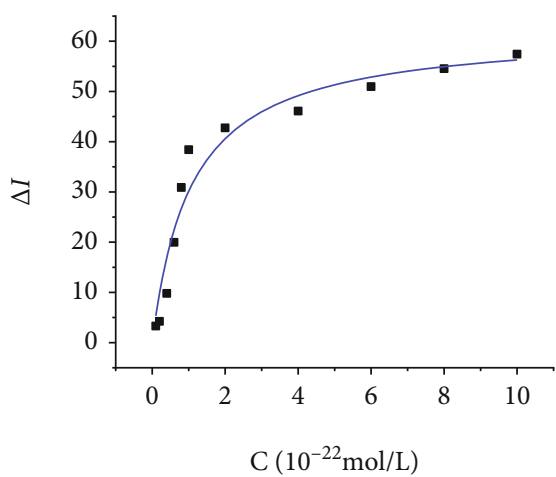

(b)

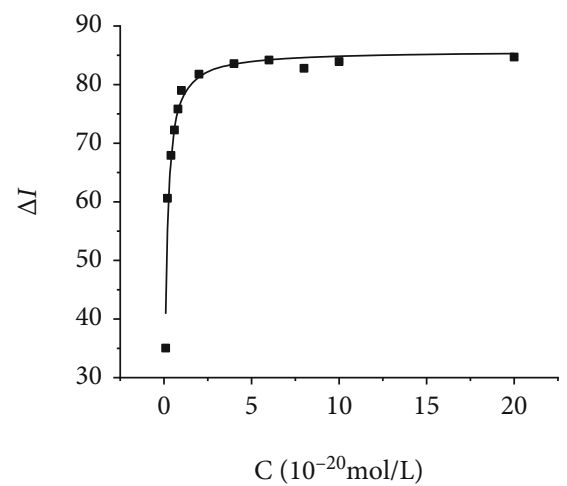

(c)

Figure 5: Current change rates and their hyperbolic fitting curves of the biosensors that were constructed with two-year-old $P$. notoginseng root tip meristems in sensing sodium nitrate at $10^{-22}-10^{-20} \mathrm{~mol} / \mathrm{L}$ (a), three-year-old $P$. notoginseng root tip meristems in sensing sodium nitrate at $10^{-23}-10^{-21} \mathrm{~mol} / \mathrm{L}(\mathrm{b})$, and four-year-old $P$. notoginseng root tip meristems in sensing sodium nitrate at $10^{-21}-10^{-19} \mathrm{~mol} / \mathrm{L}(\mathrm{c})$.

TABle 1: Hyperbolic equations of $P$. notoginseng root tip meristems of different ages interacting with sodium nitrate.

\begin{tabular}{lcr}
\hline Panax notoginseng age & Hyperbolic equation & Coefficient of determination $\left(R^{2}\right)$ \\
\hline 2-year-old & $\Delta I \%=8.2475 \times 10^{-20} \mathrm{C} /\left(0.1177+\mathrm{C} \times 10^{-21}\right)$ & 0.9860 \\
3-year-old & $\Delta I \%=6.2259 \times 10^{-21} \mathrm{C} /\left(1.0666+\mathrm{C} \times 10^{-22}\right)$ & 0.9516 \\
4-year-old & $\Delta I \%=8.5887 \times 10^{-19} \mathrm{C} /\left(0.1104+\mathrm{C} \times 10^{-22}\right)$ & 0.9684 \\
\hline
\end{tabular}

to synthesis and catabolism during the growth and development of the $P$. notoginseng plants $[38,39]$. Inorganic nitrogen (nitrate nitrogen and ammonium nitrogen) in plants can be converted into amino nitrogen via a series of reduction reactions [35]. Amino nitrogen forms amides via an initial synthesis, through which the nitrogen forms are stored as amides. The amides further form amino acids and finally accumulate as protein biomass [40].

Studies have shown that the formation of the $P$. notoginseng root system is basically completed within the first two years of growth, during which the plants absorb high amounts of nutrients to promote growth, which mainly results in an increase in root biomass, including the expansion and thickening of the taproots and the formation of many new lateral roots [41]. At this stage, the plants mainly use inorganic nitrogen forms to synthesize biomacromolecular materials, such as amino acids and nucleotides. The increase in biomass was associated with an increase in sensing sensitivity to inorganic nitrogen nutrients, such as nitrate and urea (the sensing sensitivities to sodium nitrate and urea were $1.3445 \times 10^{-23}$ and $5.3580 \times 10^{-22} \mathrm{~mol} / \mathrm{L}$, respectively). In contrast, the root growth rate of three- and four-year-old $P$. notoginseng plants slowed down. Those plants gradually senesced, and their capability to synthesize amino acids and nucleotides using inorganic nitrogen nutrients gradually declined [42], and as a result, their sensing capability was also reduced, which was particularly obvious for the four-year-old plants (the sensing sensitivity to sodium nitrate and urea was reduced by 10 - and 100 -fold, respectively).

In addition, the capability of the $P$. notoginseng root system to sense sodium glutamate decreased initially and then increased. As a key intermediate metabolite of transamination, sodium glutamate plays an important role in amino acid metabolism. The four-year-old $P$. notoginseng plants were 


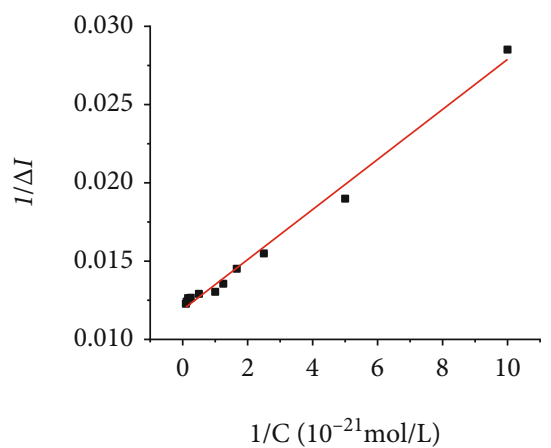

(a)

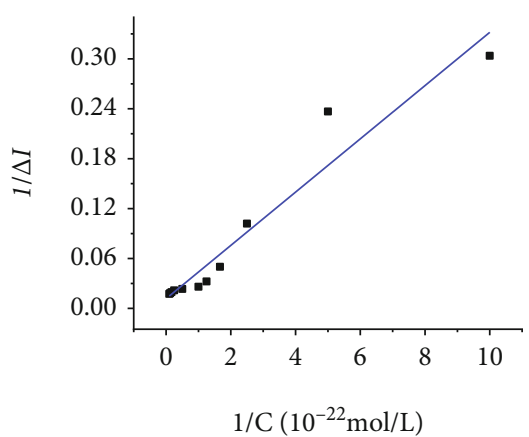

(b)

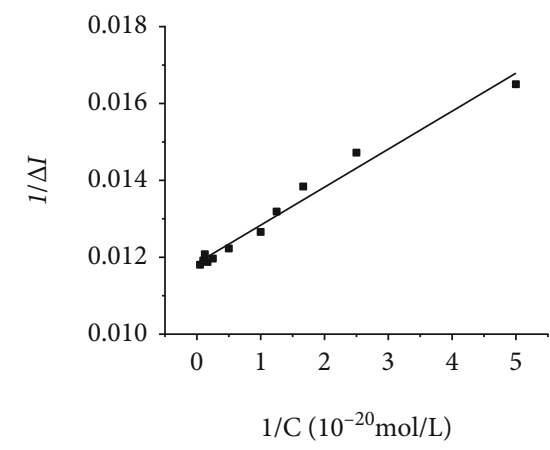

(c)

FiguRE 6: Double reciprocal-based fitting curves of the biosensors constructed with root tip meristems of two-year-old P. notoginseng (a), three-year-old P. notoginseng (b), and four-year-old P. notoginseng (c) in sensing sodium nitrate solutions.

TABLE 2: Double reciprocal linear regression equations of $P$. notoginseng root tip meristems of different ages interacting with sodium nitrate.

\begin{tabular}{lcc}
\hline Panax notoginseng age & Double reciprocal equation & Coefficient of determination $\left(\mathrm{R}^{2}\right)$ \\
\hline 2-year-old & $1 / \Delta I(\%)=0.0016 \times 10^{-21} 1 / \mathrm{C}+0.0119$ & 0.9894 \\
3-year-old & $1 / \Delta I(\%)=0.03202 \times 10^{-22} 1 / \mathrm{C}+0.01173$ & 0.9587 \\
4-year-old & $1 / \Delta I(\%)=9.88265 \times 10^{-24} 1 / \mathrm{C}+0.01185$ & 0.9764 \\
\hline
\end{tabular}

TABLE 3: Interconnected allosteric interaction constant Ka of the Panax notoginseng root tip meristem for five important nitrogen nutrients.

\begin{tabular}{lccc}
\hline Nitrogen ligand & $\begin{array}{c}\text { 2-year-old Panax notoginseng Ka } \\
\left(\mathrm{Mol} \cdot \mathrm{L}^{-1}\right)\end{array}$ & $\begin{array}{c}\text { 3-year-old Panax notoginseng Ka } \\
\left(\mathrm{Mol}^{-1} \mathrm{~L}^{-1}\right)\end{array}$ & $\begin{array}{c}\text { 4-year-old Panax notoginseng Ka } \\
\left(\mathrm{Mol} \cdot \mathrm{L}^{-1}\right)\end{array}$ \\
\hline Urea & $5.3580 \times 10^{-22}$ & $5.0750 \times 10^{-21}$ & $1.2879 \times 10^{-20}$ \\
Sodium nitrate & $1.3445 \times 10^{-23}$ & $2.7298 \times 10^{-22}$ & $8.3398 \times 10^{-22}$ \\
$\begin{array}{l}\text { Sodium } \\
\text { glutamate }\end{array}$ & $1.3595 \times 10^{-20}$ & $3.4230 \times 10^{-19}$ & $1.8912 \times 10^{-20}$ \\
$\mathrm{IMP}_{\mathrm{Na}}$ & $9.1561 \times 10^{-21}$ & $5.6638 \times 10^{-21}$ & $1.3703 \times 10^{-17}$ \\
$\mathrm{GMP}_{2} \mathrm{Na}_{2}$ & $1.5147 \times 10^{-20}$ & $1.4628 \times 10^{-20}$ & $8.5399 \times 10^{-18}$ \\
\hline
\end{tabular}

fully matured, and their demand for amino acids began to shift from biomass growth to the synthesis of antiaging proteins or enzymes $[43,44]$. At this age, the capability of the plants to sense, absorb, and transport inorganic nitrogen to form organic nitrogen was declining, which increased their capability to sense glutamate. With the increase in plant age, the change in the capability to sense disodium inosinate and disodium guanylate was basically similar. The demand of the plants for disodium inosinate and disodium guanylate was relatively constant during the first three years of growth, but decreased in the fourth year, and the sensing sensitivity decreased accordingly. Moreover, the sensitivity for sensing nucleotides was significantly lower than that for sensing inorganic nitrogen forms, indicating that the nucleotides 
TABLE 4: Comparison of various detection methods available.

\begin{tabular}{lcccc}
\hline Method & Sample & Detection target & Detection limit (Mol/L) & References \\
\hline $\begin{array}{l}\text { Liquid-membrane electrodes with } \\
\text { organotin carriers }\end{array}$ & Plasticized polyvinyl chloride membranes & Nitrate & $3.200 \times 10^{-5}$ & {$[32]$} \\
Catalytic-spectrophotometric & Water & Nitrate & $4.838 \times 10^{-4}$ & {$[33]$} \\
Fluorescence-spectroscopic & Water, soil & Nitrate & $1.000 \times 10^{-5}$ & {$[31]$} \\
$\begin{array}{l}\text { Chemiluminescence } \\
\text { Electrochemical root material sensor }\end{array}$ & Water & Nitrate & $3.200 \times 10^{-7}$ & {$[34]$} \\
\hline
\end{tabular}

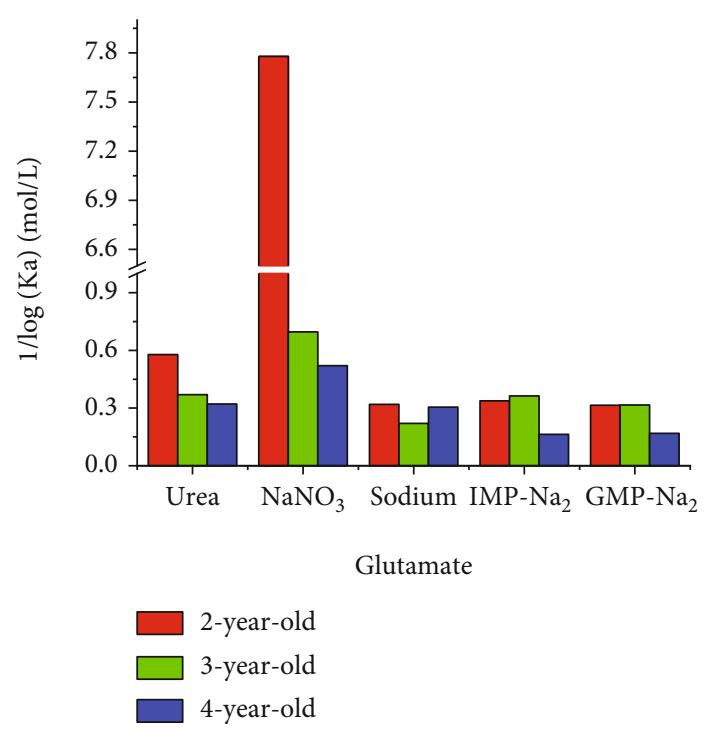

Figure 7: Comparison of the sensing sensitivity of $P$. notoginseng root tip meristems of different ages to five types of nitrogen ligands.

available in the rhizosphere had minimal effects on the plants during the growth process because nucleotides for DNA and RNA synthesis could be autotrophic and reused through the salvage pathway [45]. Therefore, the sensing sensitivity of the four-year-old plants to disodium guanylate and disodium inosinate declined rapidly, decreasing by 100 - (from 1.4628 $\times 10^{-20}$ dropped to $8.5399 \times 10^{-18}$ ) and 10,000 - (from $5.6638 \times 10^{-21}$ dropped to $1.3703 \times 10^{-17}$-) fold, respectively (Figure S3 and Table S2 in support material).

It is clear that the results generated by the root tip meristem biosensors truly reflected the demands of $P$. notoginseng of different ages for five nitrogen nutrients as well as the regularity in plant growth, development, and senescence. The results are also in accordance with the rationale that three years after planting, P. notoginseng can be used medicinally [45].

\subsection{Stability of the P. notoginseng Root Tip Meristem} Biosensor. The three-year-old P. notoginseng root tip meristem biosensors were kept moist and stored in a refrigerator at $4^{\circ} \mathrm{C}$ and were used every $24 \mathrm{~h}$ to determine the same concentration of urea solutions. The response current value of the biosensor was relatively stable in the first four days. The response current value on the fifth day was $51.25 \%$ of that on the first day, indicating that the root tip meristem biosen- sor could be used stably for at least four days (Table S3 in support material).

\section{Conclusions}

By constructing biosensors using $P$. notoginseng root tip meristems of different ages, we successfully quantitatively determined the capability of the plants to sense five representative nitrogen nutrients and revealed the associated kinetics of the root system in sensing the different nitrogen nutrients.

Our analysis of the kinetic parameters showed that the root tip meristem of $P$. notoginseng had the highest sensing sensitivity to sodium nitrate, indicating that sodium nitrate was the best inorganic nitrogen source for $P$. notoginseng. The sensing sensitivity of the root tip meristem of $P$. notoginseng to inorganic nitrogen nutrients decreased with age. The capability of the four-year-old plants to sense disodium inosinate and disodium guanylate was reduced by at least 100 -fold. We speculate that the decreasing demand for nucleotides with age is in accordance with the decreasing growth rate of the plants. The sensing sensitivity of the plants to nucleotides was significantly lower than that to inorganic nitrogen forms, which might be due to the fact that the nucleotides could be synthesized de novo from inorganic nitrogen sources at an early stage of plant development and could also be reused via the salvage pathway thereafter. The capability of the plants to sense sodium glutamate decreased initially and then increased as the plant aged. Three-year-old plants are mature, and their demand for accumulating biomass via transamination to synthesize biomacromolecules decreases, which might lead their decreased sensing sensitivity. Four-year-old plants enter the senescence stage, and their ability to use inorganic nitrogen to synthesize amino acids declines. However, the demand for the synthesis of proteins and enzymes related to aging resistance, such as aminotransferase for the stress response and anti-aging pathway, increases, which might result in an increase in the capacity to sense sodium glutamate.

The root tip meristem biosensors were simple to operate and had good stability: the stability test showed that the biosensor could be stable used for at least four days. The biosensor provides a new approach for studying the kinetics of plant root tips in sensing various nutrient components, the mechanisms of sensing, absorption, and transport of nutrients, and nutrient-related cell signal transduction. 


\section{Data Availability}

The data used to support the findings of this study are included within the support material file.

\section{Additional Points}

Highlights. (i) It developed a new Panax notoginseng root tip meristem biosensor and applied it to nitrogen sensing research. (ii) It determines the sensor kinetic parameters of 5 kinds of nitrogen nutrition. (iii) Sodium nitrate is the best inorganic nitrogen source for Panax notoginseng. (iv) The sensing ability of Panax notoginseng to sodium glutamate is related to anti-aging.

\section{Conflicts of Interest}

The authors declare no conflicts of interest.

\section{Authors' Contributions}

Zi Qing Zheng and Bo Niu are the co-first authors. Ding Qiang Lu and Guang Chang Pang are co-corresponding authors.

\section{Acknowledgments}

This work was supported by the National Natural Science Foundation of China [grant numbers 31901782 and 31671857].

\section{Supplementary Materials}

For comprehensive data and image analysis, please refer to Figures S1-S4 and Tables S1-S3 in the support materials. (Supplementary Materials)

\section{References}

[1] G. Krouk and T. Kiba, "Nitrogen and phosphorus interactions in plants: from agronomic to physiological and molecular insights," Current Opinion in Plant Biology, vol. 57, pp. 104109, 2020.

[2] W. H. Wang, B. Köhler, F. Q. Cao et al., "Rice DUR3 mediates high-affinity urea transport and plays an effective role in improvement of urea acquisition and utilization when expressed in Arabidopsis," The New Phytologist, vol. 193, no. 2, pp. 432-444, 2012.

[3] P. N. Pereira and J. C. Cushman, "Exploring the relationship between Crassulacean acid metabolism (CAM) and mineral nutrition with a special focus on nitrogen," International Journal of Molecular Sciences, vol. 20, no. 18, p. 4363, 2019.

[4] R. Pinton, N. Tomasi, and L. Zanin, "Molecular and physiological interactions of urea and nitrate uptake in plants," Plant Signaling \& Behavior, vol. 11, no. 1, article e1076603, 2016.

[5] L. H. Liu, U. Ludewig, W. B. Frommer, and N. von Wirén, "AtDUR3 encodes a new type of high-affinity urea/ $\mathrm{H}^{+}$symporter in Arabidopsis," The Plant Cell, vol. 15, no. 3, pp. 790-800, 2003.

[6] L. Long, Z. Yu, H. Qu et al., "Prediction of the network pharmacology-based mechanism for attenuation of athero- sclerosis in Apolipoprotein E knockout mice by Panax notoginseng saponins," Evidence-based Complementary and Alternative Medicine, vol. 2020, Article ID 8574702, 11 pages, 2020.

[7] C. H. Ho, S. H. Lin, H. C. Hu, and Y. F. Tsay, "CHL1 functions as a nitrate sensor in plants," Cell, vol. 138, no. 6, pp. 11841194, 2009.

[8] B. Hu, Z. Jiang, W. Wang et al., "Author Correction: NitrateNRT1.1B-SPX4 cascade integrates nitrogen and phosphorus signalling networks in plants," Nature Plants, vol. 5, no. 6, p. 637, 2019.

[9] C. Diaz, T. Lemaître, A. Christ et al., "Nitrogen recycling and remobilization are differentially controlled by leaf senescence and development stage in Arabidopsis under low nitrogen nutrition," Plant Physiology, vol. 147, no. 3, pp. 1437-1449, 2008.

[10] A. Q. Pham, S. H. Cho, C. T. Nguyen, and G. Stacey, "Arabidopsis Lectin receptor kinase $\mathrm{P} 2 \mathrm{~K} 2$ is a second plant receptor for extracellular ATP and contributes to innate immunity," Plant Physiology, vol. 183, no. 3, pp. 1364-1375, 2020.

[11] P. H. Wang, C. E. Lee, Y. S. Lin et al., "The glutamate receptorlike protein GLR3.7 interacts with 14-3-3 $\omega$ and participates in salt stress response in Arabidopsis thaliana," Plant Science, vol. 10, p. 1169, 2019.

[12] H. Li, X. Jiang, X. Lv et al., "Tomato GLR3.3 and GLR3.5 mediate cold acclimation-induced chilling tolerance by regulating apoplastic $\mathrm{H}_{2} \mathrm{O}_{2}$ production and redox homeostasis," Plant, Cell \& Environment, vol. 42, no. 12, pp. 3326-3339, 2019.

[13] D. Bhardwaj, A. Medici, A. Gojon, B. Lacombe, and N. Tuteja, "A new insight into root responses to external cues: paradigm shift in nutrient sensing," Plant Signaling \& Behavior, vol. 10, no. 12, article e1049791, 2015.

[14] G. W. Liu, A. L. Sun, D. Q. Li, A. Athman, M. Gilliham, and L. H. Liu, "Molecular identification and functional analysis of a maize (Zea mays) DUR3 homolog that transports urea with high affinity," Planta, vol. 241, no. 4, pp. 861-874, 2015.

[15] S. Smith and I. De Smet, "Root system architecture: insights from Arabidopsis and cereal crops," Philosophical Transactions of the Royal Society of London. Series B, Biological Sciences, vol. 367, no. 1595, pp. 1441-1452, 2012.

[16] J. S. Pate, C. A. Atkins, S. T. White, R. M. Rainbird, and K. C. Woo, "Nitrogen nutrition and xylem transport of nitrogen in ureide-producing grain legumes," Plant Physiology, vol. 65, no. 5, pp. 961-965, 1980.

[17] L. Liu, H. Shi, S. Li et al., "Integrated analysis of molybdenum nutrition and nitrate metabolism in strawberry," Frontiers in Plant Science, vol. 11, p. 1117, 2020.

[18] I. Cohen, M. Halpern, U. Yermiyahu, A. Bar-Tal, T. Gendler, and S. Rachmilevitch, " $\mathrm{CO}_{2}$ and nitrogen interaction alters root anatomy, morphology, nitrogen partitioning and photosynthetic acclimation of tomato plants," Planta, vol. 250, no. 5, pp. 1423-1432, 2019.

[19] Y. Huang, D. Lu, H. Liu et al., "Preliminary research on the receptor-ligand recognition mechanism of umami by an hT1R1 biosensor," Food \& Function, vol. 10, no. 3, pp. 12801287, 2019.

[20] Q. Xu, D. Lu, and G. Pang, "Study on Bombykol receptor selfassembly and universality of $\mathrm{G}$ protein cellular signal amplification system," ACS Sensors, vol. 4, no. 1, pp. 257-264, 2019.

[21] D. Dutta Banik, E. D. Benfey, L. E. Martin et al., "A subset of broadly responsive type III taste cells contribute to the 
detection of bitter, sweet and umami stimuli," PLoS Genetics, vol. 16, no. 8, article e1008925, 2020.

[22] A. W. Moran, K. Daly, M. A. al-Rammahi, and S. P. ShiraziBeechey, "Nutrient sensing of gut luminal environment," The Proceedings of the Nutrition Society, vol. 80, no. 1, pp. 29-36, 2020.

[23] J. Y. Zhang, Z. Cun, H. M. Wu, and J. W. Chen, "Integrated analysis on biochemical profiling and transcriptome revealed nitrogen-driven difference in accumulation of saponins in a medicinal plant Panax notoginseng," Plant Physiology and Biochemistry, vol. 154, pp. 564-580, 2020.

[24] J. Y. Zhang, Z. Cun, and J. W. Chen, "Photosynthetic performance and photosynthesis-related gene expression coordinated in a shade-tolerant species Panax notoginseng under nitrogen regimes," BMC Plant Biology, vol. 20, no. 1, p. 273, 2020.

[25] Q. Xu, D. Lu, and G. Pang, "Comparative study of hGPR120 receptor self-assembled nano-gold sensor and tissue sensor," Sensors and Actuators B Chemical, vol. 320, p. 128382, 2020.

[26] Y. Zhou, M. Ma, H. He et al., "Highly sensitive nitrite sensor based on AuNPs/RGO nanocomposites modified graphene electrochemical transistors," Biosensors \& Bioelectronics, vol. 146, p. 111751, 2019.

[27] B. Niu, L. Li, G. Pang, and D. Lu, "Construction of plant root tip mesenchymal sensors and their effects on urea sensing dynamics," Acta Agriculturae Zhejiangensis, vol. 32, no. 8, pp. 1466-1474, 2020.

[28] E. M. Zactiti and T. G. Kieckbusch, "Release of potassium sorbate from active films of sodium alginate crosslinked with calcium chloride," Packaging Technology and Science, vol. 22, no. 6, pp. 349-358, 2010.

[29] M. Reiss, A. Heibges, J. Metzger, and W. Hartmeier, "Determination of BOD-values of starch-containing waste water by a BOD- biosensor "," Biosensors \& Bioelectronics, vol. 13, no. 10, pp. 1083-1090, 1998.

[30] H. Hosseini, M. Behbahani, M. Mahyari, H. Kazerooni, A. Bagheri, and A. Shaabani, "Ordered carbohydrate-derived porous carbons immobilized gold nanoparticles as a new electrode material for electrocatalytical oxidation and determination of nicotinamide adenine dinucleotide," Biosensors \& Bioelectronics, vol. 59, pp. 412-417, 2014.

[31] Y. X. Guo, Q. F. Zhang, X. Shangguang, and G. Zhen, "Spectrofluorimetric determination of trace nitrite with ophenylenediamine enhanced by hydroxypropyl- $\beta$-cyclodextrin," Spectrochimica Acta. Part A, Molecular and Biomolecular Spectroscopy, vol. 101, pp. 107-111, 2013.

[32] V. A. Zarinskii, L. K. Shpigun, and V. M. Shkinev, Electrochemical Properties of Liquid Membranes Based on Dialkyltin (IV) Compounds in Phosphorus (V) Solutions, 1980.

[33] C. E. López Pasquali, P. Fernández Hernando, and J. S. Durand Alegría, "Spectrophotometric simultaneous determination of nitrite, nitrate and ammonium in soils by flow injection analysis," Analytica Chimica Acta, vol. 600, no. 1-2, pp. 177-182, 2007.

[34] M. Yaqoob, B. Folgado Biot, A. Nabi, and P. J. Worsfold, "Determination of nitrate and nitrite in freshwaters using flow-injection with luminol chemiluminescence detection," Luminescence, vol. 27, no. 5, pp. 419-425, 2012.

[35] J. Jiang, Z. Gai, Y. Wang et al., "Comprehensive proteome analyses of lysine acetylation in tea leaves by sensing nitrogen nutrition," BMC Genomics, vol. 19, no. 1, p. 840, 2018.
[36] R. Pandey, O. Teig-Sussholz, S. Schuster, A. Avni, and Y. Shacham-Diamand, "Integrated electrochemical Chip-onPlant functional sensor for monitoring gene expression under stress," Biosensors \& Bioelectronics, vol. 117, pp. 493-500, 2018.

[37] E. Cesewski and B. N. Johnson, "Electrochemical biosensors for pathogen detection," Biosensors \& Bioelectronics, vol. 159, p. 112214, 2020.

[38] C. Carter, S. Pan, J. Zouhar, E. L. Avila, T. Girke, and N. V. Raikhel, "The vegetative vacuole proteome ofArabidopsis thalianareveals predicted and unexpected proteins[W]," The Plant Cell, vol. 16, no. 12, pp. 3285-3303, 2004.

[39] H. Yang, J. Menz, I. Häussermann, M. Benz, T. Fujiwara, and U. Ludewig, "High and low affinity urea root uptake: involvement of NIP5;1," Plant \& Cell Physiology, vol. 56, no. 8, pp. 1588-1597, 2015.

[40] C. I. Lescano, C. Martini, C. A. González, and M. Desimone, "Allantoin accumulation mediated by allantoinase downregulation and transport by Ureide Permease 5 confers salt stress tolerance to Arabidopsis plants," Plant Molecular Biology, vol. 91, no. 4-5, pp. 581-595, 2016.

[41] M. Li, Z. Chen, J. Qian et al., "Composition and function of rhizosphere microbiome of Panax notoginseng with discrepant yields," Chinese Medicine, vol. 15, no. 1, p. 85, 2020.

[42] W. Wei, C. Ye, H. C. Huang et al., "Appropriate nitrogen application enhances saponin synthesis and growth mediated by optimizing root nutrient uptake ability," Journal of Ginseng Research, vol. 44, no. 4, pp. 627-636, 2020.

[43] L. Herzel, D. S. M. Ottoz, T. Alpert, and K. M. Neugebauer, "Splicing and transcription touch base: co-transcriptional spliceosome assembly and function," Nature Reviews. Molecular Cell Biology, vol. 18, no. 10, pp. 637-650, 2017.

[44] C. Carter, S. Pan, J. Zouhar, E. L. Avila, T. Girke, and N. V. Raikhel, "The vegetative vacuole proteome of Arabidopsis thaliana reveals predicted and unexpected proteins[W]," The Plant Cell, vol. 16, no. 12, pp. 3285-3303, 2004.

[45] P. S. Parihar and J. V. Pratap, “The L. donovani Hypoxanthineguanine phosphoribosyl transferase (HGPRT) oligomer is distinct from the human homolog," Biochemical and Biophysical Research Communications, vol. 532, no. 4, pp. 499-504, 2020. 